\title{
Theoretical Analysis of the Rectangular Defect Orientation using Magnetic Flux Leakage
}

\author{
J. Sam Alaric ${ }^{1}$, V. Suresh ${ }^{2}$, A. Abudhahir ${ }^{3}$, M. Carmel Sobia ${ }^{4}$, M. Baarkavi ${ }^{5}$ \\ ${ }^{1}$ Wollega University, Department of Electrical and Electronics Engineering, Ethiopia \\ ${ }^{3}$ VEL TECH Dr.RR \& Dr. SR Technical University, Chennai 600 062, India \\ 2,4,5 National Engineering College, Electronics and Instrumentation Engineering Department, Kovilpatti, 628503, \\ Tamilnadu, India, vseie@nec.edu.in
}

\begin{abstract}
This paper presents an approach to estimate the orientation of the rectangular defect in the ferromagnetic specimen using the magnetic flux leakage technique. Three components of the magnetic flux leakage profile, such as radial, axial, and tangential component are considered to estimate the orientation of the rectangular defect. The orientation of the rectangular defect is estimated by the proposed analytical model using MATLAB software. The results calculated by the analytical model are validated by the three-dimensional finite element analysis using COMSOL Multiphysics software. Tangential component provides better performance to estimate the orientation of the rectangular defect compared with radial and axial component of the magnetic flux leakage profile.
\end{abstract}

Keywords: Magnetic flux leakage, orientation of the defect, radial component, axial component, tangential component, finite element analysis.

\section{INTRODUCTION}

Magnetic Flux Leakage (MFL) technique is a wellestablished Non-Destructive Testing (NDT) method to detect the surface and subsurface defects in the ferromagnetic material. The basic principle of the MFL is that a specimen under inspection is magnetized near saturation under the applied magnetic field. If there is no defect on the specimen, the majority of the magnetic flux lines pass through the specimen material itself, in contrast, the leakage field is created due to the smaller permeability in the defect region [1]-[4]. Three major vector components of the leakage profile are radial, axial, and tangential. The geometry of the defect and its dimension may change the pattern of the three components [5], [6]. Forward and Inverse approach are referred to in the MFL inspection technique. Dipole based analytical model and finite element based numerical model provide the solution for a forward approach. In this work, forward approach is considered to detect the orientation of the rectangular shaped defect. In forward approach, the MFL testing is performed in the calibrated sample with a known defect and the pattern of the leakage profile is recorded. The data obtained through the in-situ inspection of the specimen in literature are compared with the MFL profile generated by the calibrated samples. Recording the pattern of the leakage profile for the known defect in forward approach is not necessary when the dipole model is considered [2], [7]. Zatsepin et al. (1966) introduced an analytical model using the point or strip of dipole for rectangular shape defect [8]. The magnitude of the leakage profile and the externally applied field are not considered. Later on, Shcherbinin et al. (1972) extended the Zatsepin model by considering the 3-D rectangular shape defect with finite size. The maximum amplitude of the radial and axial components of leakage profile in the symmetry axis was considered and the size of the defect was not discussed. [9]. Forster et al. (1986) improved the model of Shcherbinin for the same type of defect by considering the magnitude of the applied field and magnetic property of the specimen [10]. Minkov et al. (2002) applied the dipolar model to 3-D rectangular defect and reported that the strength of the radial component leakage profile is directly proportional to the depth of the defect [11]. Sushant.

Dutta et al. (2009) proposed a 3-D analytical model for surface breaking in ferromagnetic specimen which has a maximum magnitude for radial component rather than for axial and tangential. Leakage signal prediction and defect characterization were also performed using the analytical model [12]. Maryam Ravan et al. (2010) developed an analytical model for the arbitrary defect sizing. The main drawback of that method is longer computation time [13]. Wenhua Han et al. (2014) developed an algorithm to estimate the two-dimensional defect in fast manner and the length of the defect is not considered [14]. Dipole model was developed to measure the alternating magnetic field 
created for circular type defect in the titanium alloy pipe. The eddy current principle is used in this study. Distorted eddy current in the defect region generates the alternating magnetic field in the radial direction of the pipeline. Volumetric studies are performed to validate the analytical model along with the finite element method and experimental method [4]. Analytical model for the radial component of the leakage profile is proposed and lift-off study is also performed. Estimation of the defect from analytical model is correlated with the actual defect parameter. Performance of the analytical model is validated through experimental results [5]. The performance of the radial and axial component of non-orientated rectangular defect is analyzed and we conclude that the radial component provides excellent performance to estimate the length and depth of the defect [15]. COMSOL Multiphysics software is employed to estimate the crack depth using alternating current potential drop technique [16]. The effect of defect orientation of the steel plates was studied and the influence of the orientation was estimated in terms of crack propagation [17].

In general, the rectangular defect was presented with orientation on the specimen. In this research work analytical model is proposed to estimate the orientation of the rectangular defect using the three components of the leakage profile. Among the three components, tangential component provides the good performance to estimate the orientation of the defect. For validation, the finite element studies are performed by using COMSOL Multiphysics software.

\section{ANALYTICAL MODEL FOR THREE COMPONENTS OF MFL}

Fig.1. shows the rectangular shape defect on the steel slab with the notation of length ' 2 l', width ' $2 \mathrm{w}$ ', and depth 'd'. The angle between the discontinuity orientation and magnetization direction is represented by ' $\beta$ '.

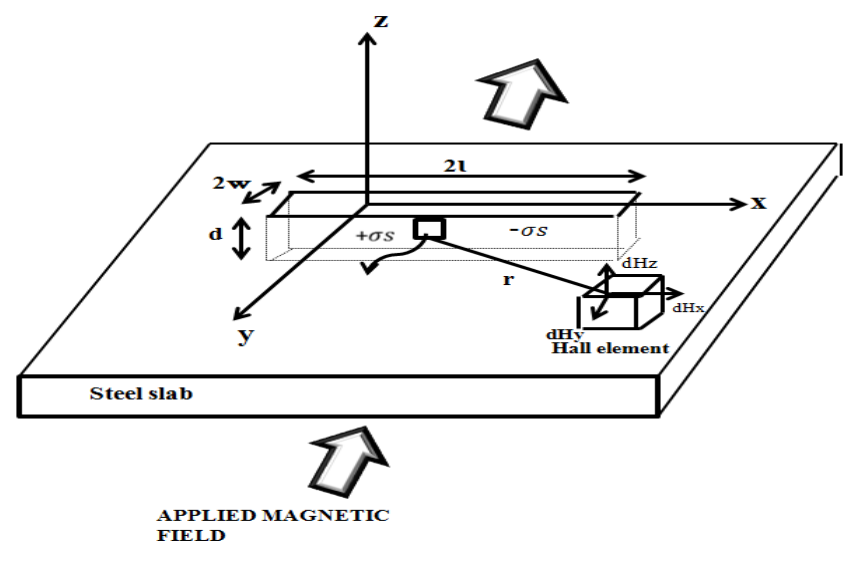

Fig.1. Dipolar representation of rectangular defect.

Three components of the leakage profile are represented as axial component ' $\mathrm{B}_{\mathrm{x}}$ ', tangential component ' $\mathrm{B}_{\mathrm{y}}$ ', and radial component ' $\mathrm{B}_{\mathrm{z}}$ '. The orientation of the defect may affect the three components of the leakage profile. The Axial ( $\left.x^{\prime}\right)$, tangential ( $\left.y^{\prime}\right)$, and radial ( $\left.\mathrm{z}^{\prime}\right)$ component of the magnetic field at a point with coordinates $\mathrm{x}^{\prime}, \mathrm{y}^{\prime}$, and $\mathrm{z}^{\prime}$ is given by

$$
\begin{aligned}
& B_{x^{\prime}}(\beta)=\frac{\sin ^{2} \beta \sigma_{m s}}{2 \pi \mu_{\mathrm{o}}} \\
& {\left[\begin{array}{c}
\arctan \frac{d\left(x^{\prime} \sin \beta+b\right)}{\left(x^{\prime} \sin \beta+b\right)^{2}+z^{\prime}\left(z^{\prime}+d\right)} \\
-\arctan \frac{(x \sin \beta-b)}{\left(x^{\prime} \sin \beta-b\right)^{2}+z^{\prime}\left(z^{\prime}+d\right)}
\end{array}\right]} \\
& B_{y^{\prime}}(\beta)=\frac{\sin \beta \cos \beta \sigma_{m s}}{2 \pi \mu_{\mathrm{o}}} \\
& {\left[\begin{array}{l}
\arctan \frac{d\left(x^{\prime} \sin \beta+b\right)}{\left(x^{\prime} \sin \beta+b\right)^{2}+z^{\prime}\left(z^{\prime}+d\right)} \\
-\arctan \frac{(x \sin \beta-b)}{\left(x^{\prime} \sin \beta-b\right)^{2}+z^{\prime}\left(z^{\prime}+d\right)}
\end{array}\right]} \\
& B_{Z^{\prime}}(\beta)=\frac{\sin \beta \sigma m s}{4 \pi \mu_{\circ}} \\
& {\left[\begin{array}{c}
\ln \frac{\left(x^{\prime} \sin \beta+b\right)^{2}+\left(z^{\prime}+d\right)^{2}}{\left(x^{\prime} \sin \beta-b\right)^{2}+\left(z^{\prime}+d\right)^{2}} \\
+\ln \frac{\left(x^{\prime} \sin \beta-b\right)^{2}+z^{\prime 2}}{\left(x^{\prime} \sin \beta+b\right)^{2}+z^{\prime 2}}
\end{array}\right]}
\end{aligned}
$$

To analyze the MFL signal characteristics of the discontinuity in a different orientation, the length 21 , the width $2 \mathrm{w}$, depth $\mathrm{d}$, lift-off $\mathrm{z}^{\prime}$, and $\sigma_{\mathrm{ms}} / 4 \pi \mu$ were assumed to be $2 \mathrm{~cm}, 1.5 \mathrm{~cm}, 0.50 \mathrm{~cm}, 1 \mathrm{~mm}$ and 1 , respectively. The $\mathrm{B}_{\mathrm{x}}(\beta), \mathrm{B}_{\mathrm{y}^{\prime}}(\beta)$, and $\mathrm{B}_{\mathrm{z}}(\beta)$ are calculated for the orientation angle $\beta$ from 0 to 90 degrees.

From Fig.2. it is observed that the magnitude of the three components of the MFL signal varied for the different orientations of the defect. The defect orientation angle $\beta$ is increased from 0 to 90 degrees and the analytical model output is observed. The amplitude of the axial and radial component is increased and very minor magnitude changes in the 75, 80 and 90-degree orientation are observed. The amplitude of the tangential component is first increased from 0 to 45-degree orientation and then decreased from 45 to 90 degrees. Polynomial expression for axial, radial, and tangential component of the leakage profile is derived using the magnitude of the leakage profile. Tangential component has two polynomial expressions, the expression (6a) is used to estimate the orientation from 0 to 45 degrees and the expression (6b) is used to estimate the orientation from 46 to 90 degrees.

$$
\begin{aligned}
& \text { axial angle }=33 B_{x}^{3}-69 B_{x}^{2}+107 B_{x}+3 \\
& \text { radial angle }=2 B_{z}^{3}-6 B_{z}^{2}+21 B_{z}+1 \\
& \text { tangential angle }=-242 B_{y}^{3}+286 B_{y}-74 \\
& \text { tangential angle }=222 B_{y}^{3}+286 B_{y}^{2}-28 B_{y}+90
\end{aligned}
$$




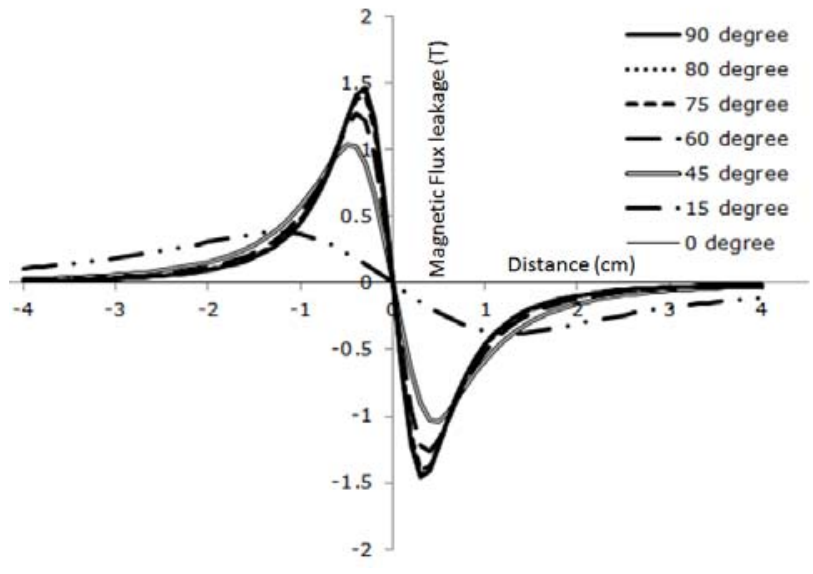

a)

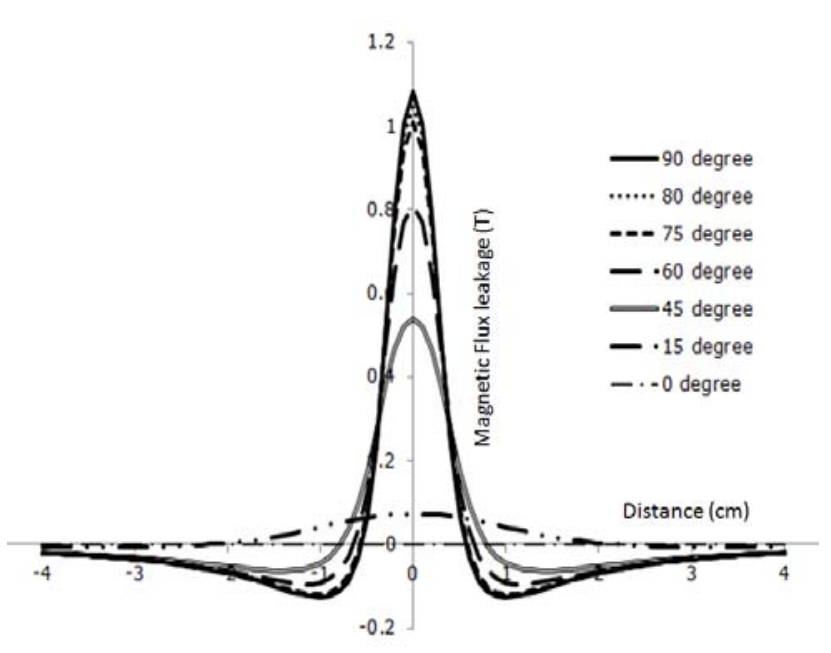

b)

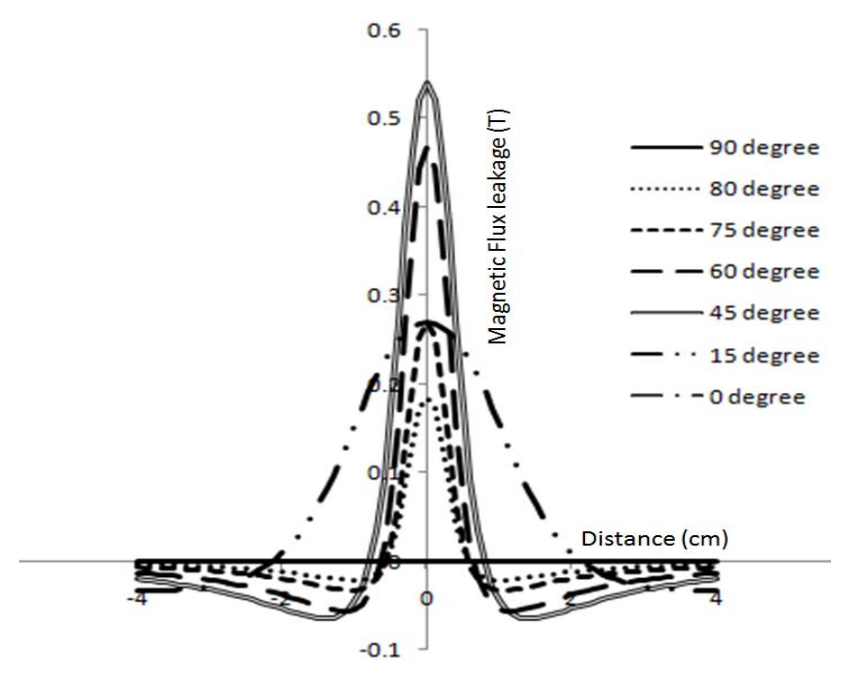

c)

Fig.2. MFL profile of analytical model output for the different orientation of discontinuity

a) Axial profile, b) radial profile, c) tangential profile.
Error percentage of the three components of the MFL profile during the estimation of the angle is shown in Table 1. The average error percentage of the radial and axial leakage profile component is ' $13^{\text {' }}$ and ' 7 '. When comparing axial and radial component, the axial component has less average error percentage. It is observed that the radial component failed to estimate the orientation of the defect. Among the three components, tangential leakage profile provides better estimation. Axial and tangential components are considered to estimate the defect orientation in numerical studies.

Table 1. Estimated error \% for orientation of three profiles.

\begin{tabular}{|c|c|c|c|c|c|c|}
\hline 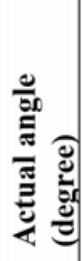 & 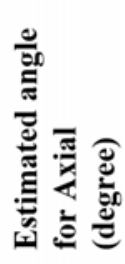 & 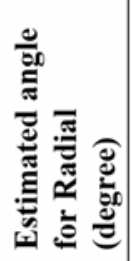 & 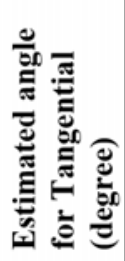 & 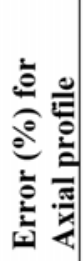 & 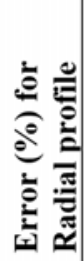 & 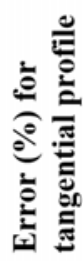 \\
\hline 15 & 10 & 17 & 15 & 33 & 46 & 0 \\
\hline 45 & 47 & 39 & 45 & 4 & 13 & 0 \\
\hline 60 & 60 & 67 & 60 & 0 & 11 & 0 \\
\hline 75 & 71 & 78 & 75 & 5 & 4 & 0 \\
\hline 80 & 81 & 79 & 80 & 1 & 1 & 0 \\
\hline 90 & 89 & 82 & 90 & 1 & 8 & 0 \\
\hline
\end{tabular}

\section{NUMERICAL MODEL FOR TANGENTIAL AND AXIAL COMPONENT OF MFL}

The finite element approach is an efficient method to identify the defect on the inspected specimen. Threedimensional finite element studies are performed to validate the proposed analytical model. COMSOL Mutiphysics 4.3a modeling software is utilized to implement the finite element analysis. AC/DC module of the software is facilitated to compute the electric and magnetic field analysis on the inspected specimen. Fig.3. shows the threedimensional geometry of 15-degree oriented rectangular defect on the rectangular ferromagnetic steel slap with permanent magnet. The length, width and thickness of the ferromagnetic steel slab are $100 \mathrm{~cm} \times 100 \mathrm{~cm} \times 15 \mathrm{~cm}$. The rectangular defect is of length $50 \mathrm{~cm}$, width $5 \mathrm{~cm}$ and depth $7.5 \mathrm{~cm}$ with 15,45 and 80-degree orientations. Two permanent magnets are used to magnetize the inspected specimen. The entire arrangement is surrounded by the air bounded domain.

Table 2. shows the domain specification of ferromagnetic plate and the permanent magnet in COMSOL Multiphysics 4.3a.

In the preprocessing of the geometry, tetrahedral element is considered for meshing operation. Fig.4. shows the tetrahedral meshed geometry of the specimen with the help of 131,338 elements and 838,650 numbers of degrees of freedom. Computation time taken for this preprocessing is 72 seconds. 


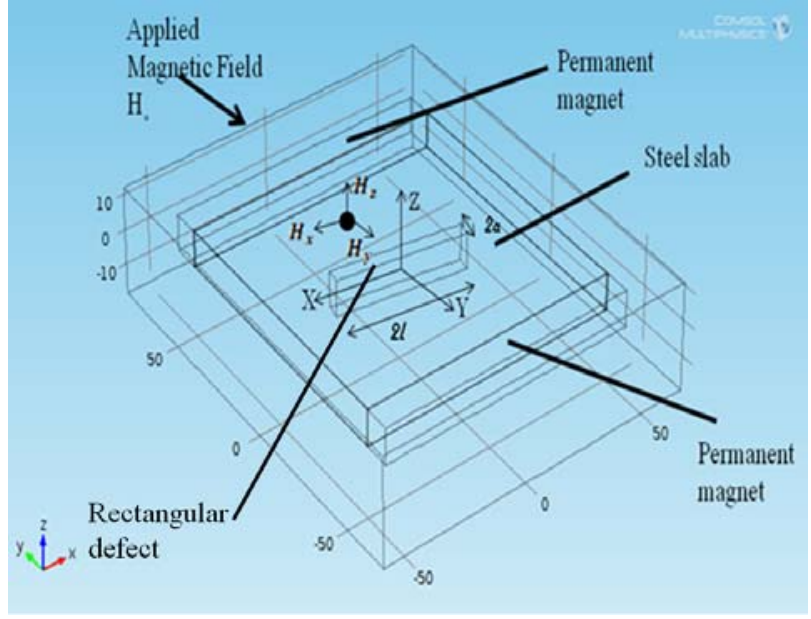

Fig.3. Geometry of the specimen with defect.

Table 2. Domain specification of the model.

\begin{tabular}{|c|c|c|}
\hline Specification & Ferromagnetic Plate & $\begin{array}{c}\text { Permanent } \\
\text { Magnet }\end{array}$ \\
\hline Material & $\begin{array}{c}\text { ASTM A213-76a, } \\
\text { Grade T9 (9Cr-1Mo) }\end{array}$ & Neodymium \\
\hline $\begin{array}{c}\text { Relative } \\
\text { permittivity }\end{array}$ & 1 & 1 \\
\hline $\begin{array}{c}\text { Electrical } \\
\text { conductivity } \\
{[\mathrm{S} / \mathrm{m}]}\end{array}$ & $4.032 \mathrm{e} 6$ & $\begin{array}{c}\text { sigma_solid_1 } \\
(\mathrm{T}[1 / \mathrm{k}])\end{array}$ \\
\hline $\begin{array}{c}\text { Relative } \\
\text { permeability }\end{array}$ & 100 & 4000 \\
\hline
\end{tabular}

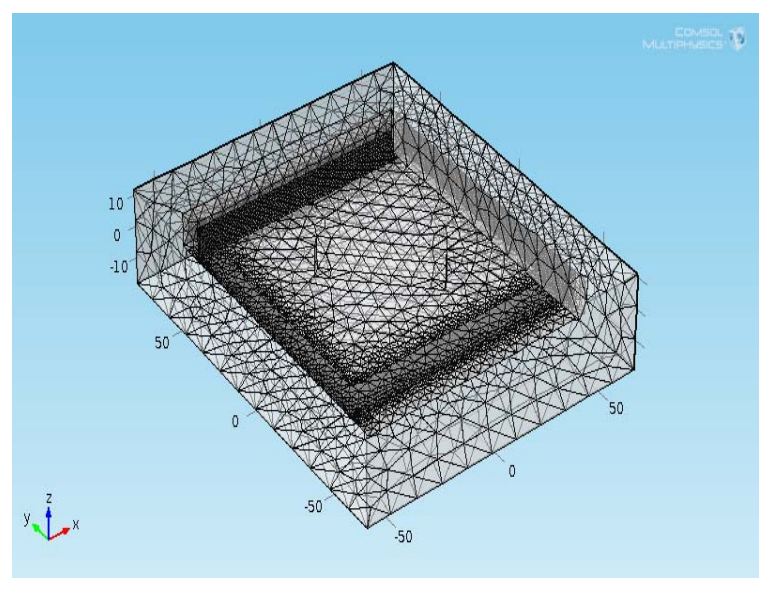

Fig.4. Meshed geometry of the specimen with defect.

The property of the mesh considered for this finite element analysis is shown in Table 3.

Table 3. Properties of mesh.

\begin{tabular}{|c|c|}
\hline Element size parameter & Value \\
\hline Max element size & 12 \\
\hline Min element size & 1.5 \\
\hline Max element growth rate & 1.45 \\
\hline Resolution of curvature & 0.5 \\
\hline Resolution of narrow regions & 0.6 \\
\hline
\end{tabular}

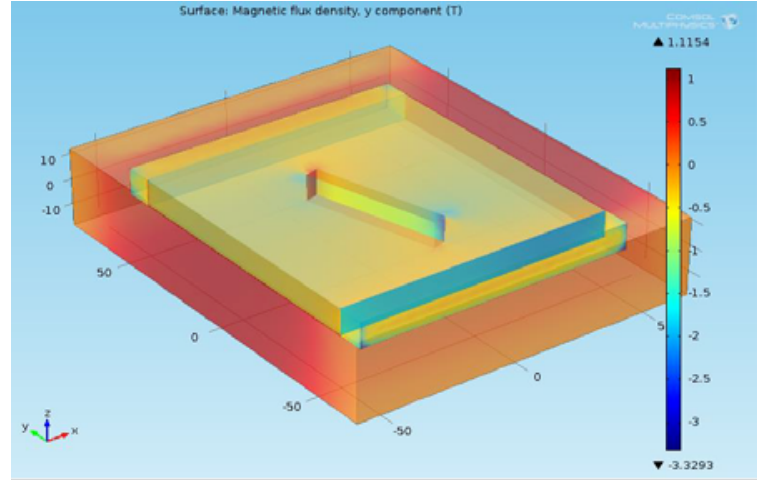

a) 15-degree orientation

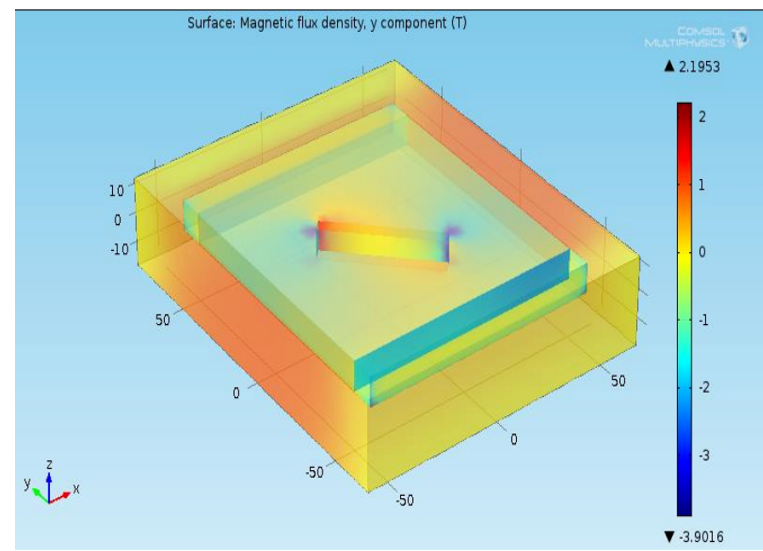

b) 45-degree orientation

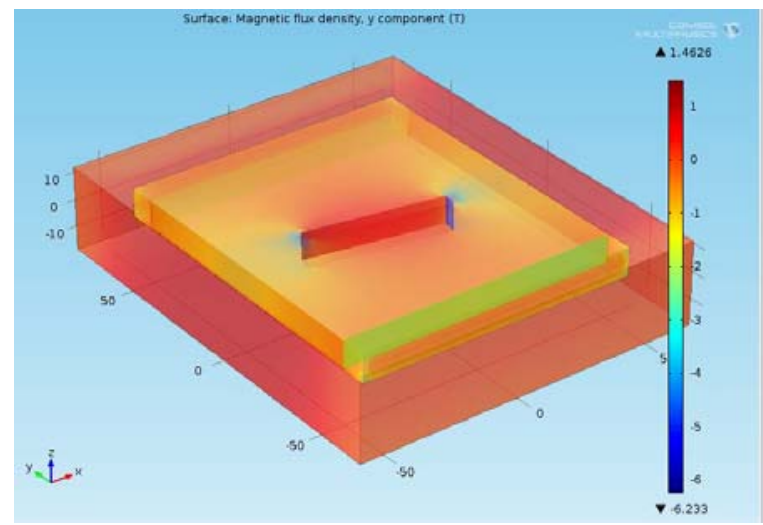

c) 80-degree orientation

Fig.5. Surface plots of the tangential component for different orientations of the defect.

In the $\mathrm{AC} / \mathrm{DC}$ module of COMSOL Multiphysics software, the stationary solver study is performed on the developed geometry. In post processing of the solved geometry the surface plot is constructed. Fig.5. shows the surface plot of 15,45 and 80-degree orientation of the defect. The uniform distribution of the magnetic field in the entire region of the specimen and the variation of the leakage profile in the defect region shows the effective arrangement of permanent magnet along with the inspected specimen. The stepwise single point line scan is performed for $100 \mathrm{~cm}$ length of the specimen and the magnitude of the axial and tangential component of the leakage profile is observed. 


\section{RESULTS AND DISCUSSION}

To validate the proposed analytical model a comparison study is performed between the outputs of analytical model with numerical model. The dimension of the defect in the comparison study is considered to be of length $50 \mathrm{~cm}$, width $5 \mathrm{~cm}$, and depth $7.5 \mathrm{~cm}$. The orientation of the defect with respect to the applied magnetic field is 15 degrees, 45 degrees, and 80 degrees. Fig.6. shows the normalized output of the axial and tangential component of the analytical model. Normalization of the magnetic leakage profile output does not affect the defect information [2]. The magnitude of the axial component decreases while the orientation of the defect increases. In the case of tangential component, the magnitude is increased from 0 to 45 degrees of orientation and then the magnitude of the profile is decreased up to 90 degrees of orientation.

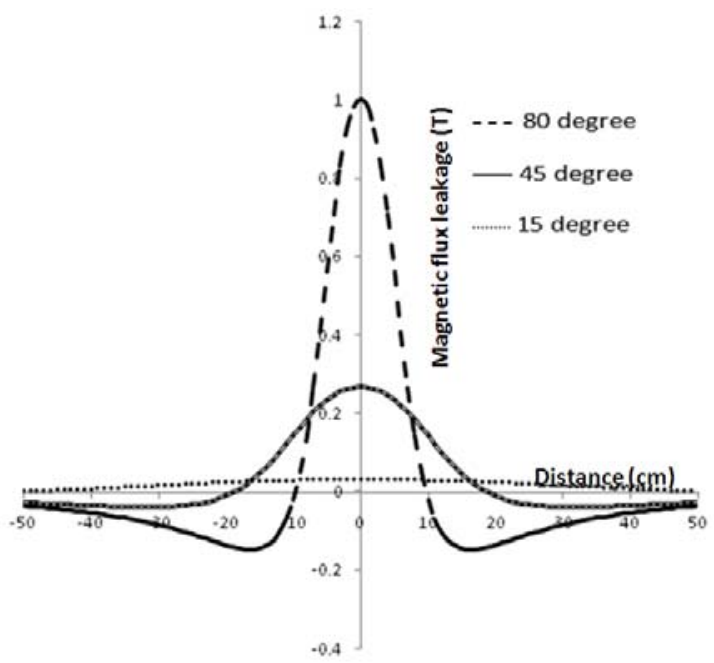

a)

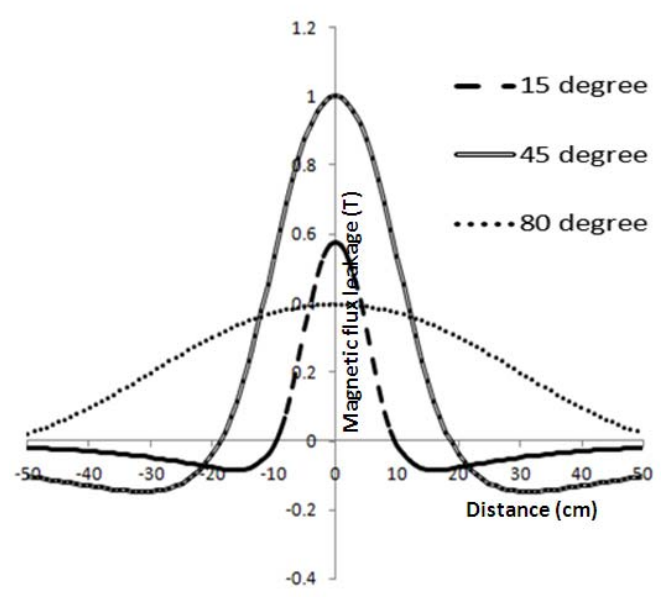

b)

Fig.6. Analytical output for different orientation a) axial component, b) tangential component.

The polynomial expression is derived using the actual angle with the corresponding analytical output magnitude of the leakage field. The equation (7) is derived with the help of axial profile magnitude and the equation (8) is derived with the help of tangential profile magnitude. Error percentage of the angle estimation for the axial and tangential component of the analytical model output is shown in Table 4.

$$
\begin{aligned}
& \text { axial angle }=-145 B_{x}^{3}+208 B_{x}^{2}+24 B_{x}-1 \\
& \text { tangential angle }=B_{y}^{3}+29 B_{y}^{2}-8 B_{y}+1 \\
& \text { tangential angle }=-238 B_{y}^{3}+182 B_{y}^{2}-74 B_{y}+90
\end{aligned}
$$

\begin{tabular}{|c|c|c|c|c|}
\hline 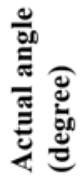 & 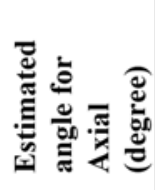 & 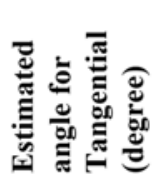 & 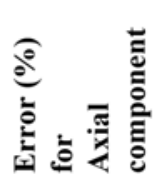 & 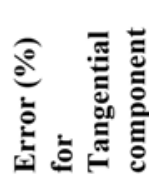 \\
\hline 15 & 21 & 15 & 28 & 0 \\
\hline 45 & 41 & 45 & 8 & 0 \\
\hline 80 & 82 & 80 & 2 & 0 \\
\hline
\end{tabular}

Table 4. Estimated error \% for analytical model.

Fig.7. shows the normalized output of the axial and tangential component of numerical model. The magnitude of the leakage profile of both components in numerical model is varied as discussed in the analytical model output. The polynomial expression is derived using the actual angle with the corresponding numerical output magnitude of the leakage field.

$$
\begin{aligned}
\text { axial angle } & =9 B_{x}^{3}-46 B_{x}^{2}+79 B_{x}-2 \\
\text { tangential angle } & =67 B_{y}^{3}-88 B_{y}^{2}+67 B_{y}+0 \\
\text { tangential angle } & =-39 B_{y}^{3}-12 B_{y}^{2}-53 B_{y}+90
\end{aligned}
$$

\begin{tabular}{|c|c|c|c|c|}
\hline 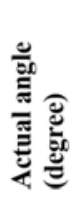 & 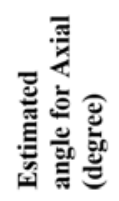 & 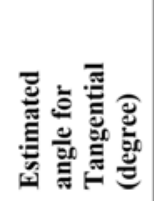 & 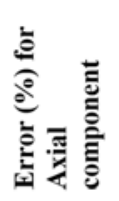 & 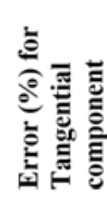 \\
\hline 15 & 18 & 15 & 20 & 0 \\
\hline 45 & 42 & 45 & 5 & 0 \\
\hline 80 & 83 & 80 & 3 & 0 \\
\hline
\end{tabular}

Error percentage of the angle estimation for the axial and tangential component of the numerical model output is shown in Table 5 .

Table 5. Estimated error \% for numerical model.

The tangential component provides the minimum error percentage when compared to the axial component in analytical and numerical model output. The orientation of the defect on the ferromagnetic specimen is estimated accurately using the tangential component of the MFL profile. 


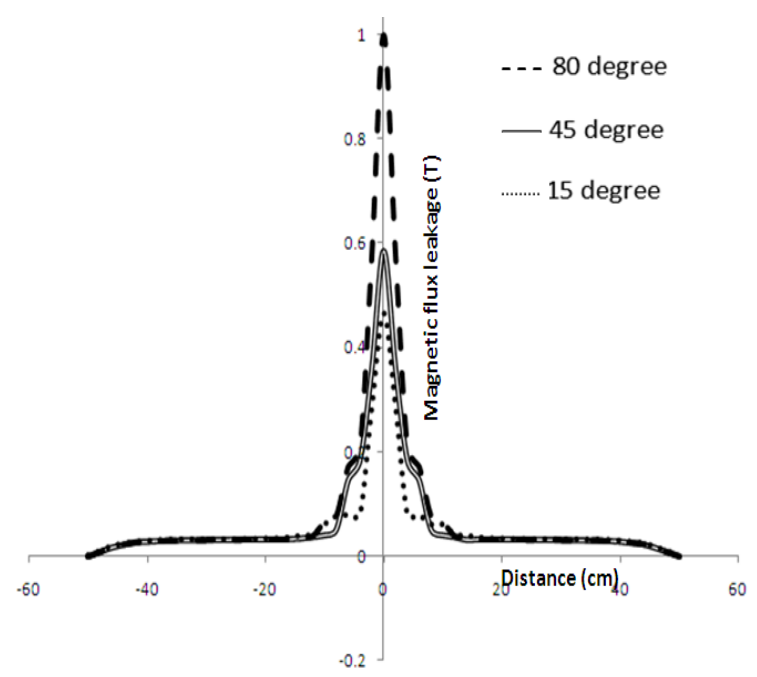

a)

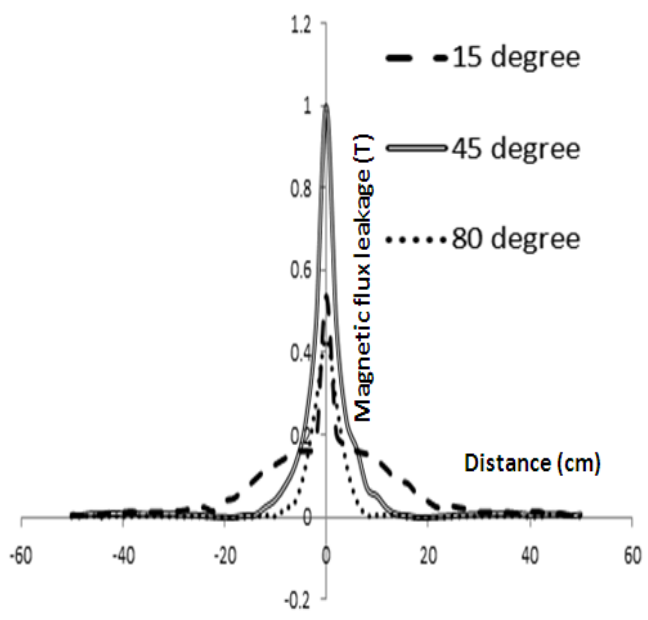

b)

Fig.7. Numerical output for different orientation a) axial component, b) tangential component.

\section{CONCLUSION}

We have developed an analytical model to estimate the orientation of the defect in ferromagnetic specimen. The result of the analytical model analysis showed that the radial component has large error to estimate the angle of the defect, and hence, the axial and tangential components are considered in the numerical study. The tangential component measured the defect orientation in precise manner when compared to the axial component. For validation purpose the numerical studies are performed using COMSOL Multiphysics software. Good correlation among the analytical and numerical studies revealed the good performance of the proposed method. The most important step of the forward approach, such as recording the pattern of the leakage profile for the known defect, is eliminated by using the proposed analytical model and also the proposed model provides support to reconstruct the defect in the inverse MFL problem.

\section{ACKNOWLEDGMENT}

The authors would like to thank Prof. Jackson Daniel for technical discussion and also thank Prof. Shankar for language correction.

\section{REFERENCES}

[1] Rao, B.P.C. (2012). Magnetic flux leakage. Journal of Non - Destructive Testing \& Evaluation, 11 (3), 7-17.

[2] Mandache, C., Clapham, L. (2003). A modelfor magnetic flux leakage signal predication. Journal of Physics D: Applied Physics, 36, 2427-2431.

[3] Jianbo, W., Yanhua, S., Yihua, K., Yun, Y., (2015). Theoritical analyses of MFL signal affected by discontinuity orientation and sensor scanning direction. IEEE Transactions on Magnetics, 51 (1).

[4] Le, M., Lee, J., Junc, J., Kima, J. (2013). Estimation of sizes of cracks on pipes in nuclear power plants using dipole moment and finite element methods. NDT \& E International, 58, 56-63.

[5] Suresh, V., Abudhahir, A., (2016). An analytical model for prediction of magnetic flux leakage from surface defects in ferromagnetic tubes. Measurement Science Review, 16 (1), 8-13.

[6] Suresh, V., Abudhahir, A., Jackson, D., (2017). Development of magnetic flux leakage measuring system for detection of defect in small diameter steam generator tube. Measurement, 95, 273-279.

[7] Mandache, C., Shiari, B., Clapham, L. (2005). Defect separation considerations in magnetic flux leakage inspection. Insight, 47 (5), 269-273.

[8] Zatsepin, N.N., Shcherbinin, V.E. (1966). Calculation of the magnetostatic field of surface defects. Defektoskopiya, 5, 50-59.

[9] Shcherbinin, V.E., Pashagin, A. (1972). Influence of the extension of a defect on the magnitude of its magnetic fields. Defektoskopiya, 8, 72-83.

[10] Forster, F. (1986). New findings in the field of non destructive magnetic leakage field inspection. NDT International, 19 (1), 3-14.

[11] Minkov, D., Takeda, Y., Shoji, T., Lee, J. (2002). Estimating the sizes of surface cracks based on Hall element measurements of the leakage magnetic field and a dipole model of a crack. Applied Physics A, 74 (2), 169-176.

[12] Dutta, S.M., Ghorbel, F.H., Stanley, R.K. (2009). Dipole modeling of magnetic flux leakage. IEEE Transactions on Magnetics, 45 (4), 1959-1965.

[13] Ravan, M, Amineh, R.K, Koziel, S, Nikolova, N.K., Reilly, J.P. (2010). Sizing of 3-D arbitrary defects using magnetic flux leakage measurements. IEEE Transactions on Magnetics, 46 (4), 1024-1033.

[14] Han, W., Shen, X., Xu, J., Wang, P., Tian, G., Wu, Z. (2014). Fast estimation of defect profiles from the magnetic flux leakage signal based on a multi-power affine projection algorithm. Sensors, 14 (9), 1645416466. 
[15] Suresh, V., Abudhahir, A. (2016). Dipole model to predict the rectangular defect on ferromagnetic pipe. Journal of Magnetics, 21 (3), 437-441.

[16] Yuting, L., Fangji, G., Zhengjun, W., Junbi, L., Wenqiang, L. (2015). Novel method for sizing metallic bottom crack depth using multi-frequency alternating current potential drop technique. Measurement Science Review, 15 (5), 268-273.
[17] Aljabar, N.J., Zhao, X.L., Al-Mahaidi, R., Ghafoori, E., Motavalli, M., Powers, N. (2016). Effect of crack orientation on fatigue behavior of CFRP-strengthened steel plates. Composite Structures, 152, 295-305.

Received November 16, 2017. Accepted February 15, 2018. 